\title{
"Maternal Birth Injury" Case Report
}

\author{
Busreea RA ${ }^{1}$, Jahan $\mathrm{A}^{2}$, Begum $\mathrm{F}^{3}$, Siddika $\mathrm{M}^{4}$, Siddiquee $\mathrm{FS}^{5}$
}

\begin{abstract}
Injury to the birth canal following vaginal delivery is not an uncommon condition. It causes PPH. Early detection of injury site and control of bleeding is an emergency. Delay detection of injury may causes the maternal morbidity and or mortality as well as. Here we presents three cases of birth canal injury following vaginal delivery. We managed those patient's accordingly.
\end{abstract}

CBMJ 2017 January: Vol. 06 No. 01 P: 31-33

Key Wards: Pregnancy, Birth trauma, Colporrhaxis, Dehisence, Vault rupture, Post natal care.

\section{Introduction}

Complications of pregnancy and childbirth are among the leading causes of death and illness for women around the world. Most maternal deaths and disabilities occur in developing countries, especially among women who are poor, uneducated or live in rural areas. Maternal injuries following childbirth are quite common and contribute significantly to maternal morbidity and even to death. Prevention, early and prompt and effective management not only minimize the morbidity but prevent many a gynaecological problems from developing later in life.

\section{Cases report-1:}

Mrs. Tahmina, Primi gravida 28 years old lady coming from Churkhai, Mymensingh was admitted in Community Based Medical College Hospital Bangladesh at her 39 weeks of pregnancy with labour pain. Her labour was started spontaneously at home 12 hours prior to admission. She received inj. Oxytocin in saline at home by village doctor. She was not in regular antenatal care. Her pregnancy was uneventful. Her labour was prolonged so she admitted in CBMCHB for better management. On admission her pulse: 92/m, BP: 100/60 $\mathrm{mm}$ of $\mathrm{Hg}$. Temp: $99^{\circ} \mathrm{F}$.

Per abdominal examination revealed term pregnancy, vertex presentation, longitudinal lie and fetal heart rate- $148 / \mathrm{m}$, regular, vaginal examination revealed cervical os was $05-10$ $\mathrm{cm}$ dilated, cervix-100 percent effaced, station $(-1)$, caput $(++)$ membrane ruptured, liquor deeply meconium stained. After 1 hour of admission with the help of episiotomy a severely asphyxiated weighing $3.5 \mathrm{~kg}$ was delivered. But patient developed post-partum haemorrhage (PPH) following delivery. Inspite of good uterine contraction severe per-vaginal bleeding was continuing. So causes of PPH was checking and a rent was found in the

*1. Dr. Reeva Aireen Busreea

Assistant Professor

Department of Gynae and Obs

Community Based Medical College Bangladesh Winnerpar, Mymensingh.

2. Dr. Akter Jahan

Assistant Professor

Department of Gynae and Obs

Community Based Medical College Bangladesh

Winnerpar, Mymensingh.

3. Dr. Ferdousi Begum

Assistant Professor

Department of Gynae and Obs

Community Based Medical College Bangladesh Winnerpar, Mymensingh.

4. Dr. Mohosina Siddika

Assistant Professor

Department of Gynae and Obs

Community Based Medical College Bangladesh Winnerpar, Mymensingh.

5. Dr. Fayela Sabrun Siddiquee

Registrar

Department of Gynae and Obs

Community Based Medical College Hospital

Bangladesh, Winnerpar, Mymensingh.

\author{
*Address of correspondence \\ Mobile: 01711232087 \\ E-mail :dr.reeva.islam@gmail.com
}


posterior fornix of vagina. Fatty structures were coming out through the colporrhaxis. Then she was diagnosed as a case of ruptured posterior fornix. Immediately trying to repair the posterior fornix vaginally under general anaesthesia. Inspite of tendelenberg position of patient repair was not possible due to coming out of gut and omentum through the rent of fornix of vagina, So laparotomy was done and rent was repaired by chromic catgut. She took 3 units of blood. She was discharged on $8^{\text {th }}$ day of puerperium without baby because baby was expired on $3^{\text {rd }}$ day due to birth asphyxia.

\section{Case report-2:}

Mrs. Asma, 30 years lady belonging to below average socioeconomic status, P: $4+0$,

house wife was admitted in $\mathrm{CBMCHB}$ with the $\mathrm{H} / \mathrm{O}$ home delivery 13 days back. The delivery was conducted by a traditional birth attendent. She delivered a healthy male baby. According to patients opinion she was not in regular antenatal care and she was not immunized against tetanus. Following delivery she gradually developed severe lower abdominal pain and difficulty in micturition. She also had difficulty in sitting and she became pale. For pain \& weakness she took several drugs by the help of local doctor. But gradually she became more sick and developed difficulty in walking. During admission her pulse was $96 / \mathrm{min}$, BP-100/60, Anameia (++). Abdominal examination revealed abdomen was soft but a mass was felt in left iliac fossa, which was about $12 \mathrm{~cm} \times 10 \mathrm{~cm}$ in size, the mass was firm in consistency, surface was smooth, fixed with under lying structure \& tender. On vaginal examination revealed anterior and left fornix was bulged. Uterus bulky, slight vaginal bleeding was present. Sonological report showed a large complex mass about $11 \mathrm{~cm}$. $10 \mathrm{~cm}$, solid and cystic component in the left adnexal region may be ovarian origin, pouch of douglus was clear. After laparotomy a big broad ligament haematoma was found on left side. Origin of haematoma was difficult to trace. The lower margin also could not be traced. After tracing the ureter, reasonable haemostasis was achieved and abdomen was closed after putting a drain. She took 2 units of fresh whole human blood. Her postoperative period was uneventful. On $8^{\text {th }}$ postoperative day she was discharged.

\section{Case report-3:}

Mrs. Nasrin Akhter, 30 years old lady P-2+0, hailing from Valuka, Mymensingh was admitted in $\mathrm{CBMCHB}$ with severe per vaginal bleeding, 3 hours after vaginal delivery at home. She was not in antenatal care. Her pregnancy was uneventful. On admission she was severely anaemic, pulse $120 / \mathrm{min}$, low volume, BP-80/40, skin cold and clamy. On abdominal examination height of uterus about 26 wks, which was flaccid. On vaginal examination huge amount of blood clots removed from vagina, os was open and placenta felt through os. A large swelling also felt in right lateral vaginal wall. After quick resuscitation placenta was removed manually under general anesthesia and uterine massage was done, after giving her 10 units of Syntocinon intravenously and adding 40 units in Ringer's Lactate, one thousand micrograms of Misoprostol tablet were placed in rectum. ${ }^{1}$ A big haematoma also found in the right lateral vaginal wall. Huge amount (about 2 fist) of blood clot was removed by giving incision over the vaginal wall and reasonable haemostasis was achieved after giving a drain tube into the haematoma site, vaginal packing was done. As there was no collection in the drain tube so tube and vaginal pack were removed after 24 hours. She took 3 units of fresh blood and she was discharged on $5^{\text {th }}$ postoperative day with a healthy baby.

\section{Discussion}

A pregnancy usually ends by an uncomplicated delivery in most of the cases, but may have some complications. The study of the cases of life threatening conditions will give us a way of evaluating maternal health services and also this act as a case control for maternal death cases. Birth canal injury following vaginal delivery must be excluded if patient complaints of pelvic pain, excessive per vaginal bleeding, urinary complaints, associated with anaemia. The treatment can be conservative or surgical. 
Laceration of vulval skin specially posteriorly, para urethral injury are most common. Minor perineal injury is usually as a result of mismanagement of $2^{\text {nd }}$ stage of labour. Vaginal laceration or tear usually seen following instrumental manipulative delivery. Vault also may rupture. It may be primary where only vault is involved and in secondary where cervical tear is involved. Cervical tear is rare. It is commonest cause of traumatic PPH. Minor degree left lateral tear is common during $1^{\text {st }}$ delivery. Pelvic haematoma is rare. Which usually occurs due to improper haemostasis during repair of vaginal or perineal tear and episiotomy wound. ${ }^{2}$ Para genital haematomas are of two types. Supralevator (above the levator ani muscels) and infralevator. Supralevator haematomas spread upwards and outwards beneath the broad ligament. About a third follows spontaneous vaginal delivery, caesarean section and forceps delivery. ${ }^{3}$ Fifty percent of these are recognised immediately while rest are discovered after about 24 hours. The symptoms and treatment of puerperal genital hematomas are veriable according to size, location, rate of haematoma formation and severity of patient. ${ }^{4}$ Resuscitation, volume replacement and surgical exploration are the very steps in its management. For broad ligament haematoma conservative management is recomended. ${ }^{5}$ Our second case was similar to the case described by Chia and Huang. ${ }^{6}$ Spontaneous rupture of vagina during labour is a rare condition. The term colporrhaxis describe this obstetric injury and it is subdivided into primary and secondary, spontaneous or traumatic, complete or incomplete. The condition has been associated with high parity, but it has been reported in primi gravida. ${ }^{7}$ It can occur spontaneously or following trauma or vaginal instrumentation or any event that increase intra abdominal pressure. ${ }^{8}$ Colporrhaxis reported previously $8-48 \%$ following intercourse and $16-30 \%$ reported defecation or valsalva (sneezing coughing) as precipitating event. ${ }^{9}$ Precipitated labour and use of inj Oxytocics in labour is also the factor. ${ }^{(7,10)}$

\section{Conclusion}

Almost all life-threatening complications can be prevented or treated if women have access to high-quality and appropriate health care during pregnancy, abortion, childbirth and immediately afterwards. A skilled provider, when properly equipped and supported, can provide critical life-saving interventions to avert the major causes of maternal death and disability. We should be more careful in our practice to reduce maternal morbidity and mortality. So we need to take some steps:

1. Strengthening of antenatal care visit.

2. Identifying high risk mother.

3. Counseling them for hospital delivery.

4. Identify intrapartum complication and early management or referral.

5. Strengthening of postnatal care visit.

\section{References}

1. Protocols for the management of obstetric complications, UNICEF 2003;33.

2. Dutta DC. Injuries to the Birth Canal. In; Text book of Obse. of the $8^{\text {th }}$ edition.

3. Edmonds, D.Keith (Ed), Dewhurst's Textbook of Obstetrics and Gynaecology, 6th ed, London: Blackwell Science 1999;327.

4. Bienstman-Pailleux J, Huissoud C, Dubernard G, Rudigoz RC, Management of puerperal hematomas, J Gynecol Obstet Biol Reprod (Paris) 2009;38:203-8.

5. Chohan A, Fundamentals of Obstetrics, 1st ed, Lahore: M A R Publishers, 2005;377.

6. Chia CC, Huang SC, Postpartum hemorrhage of genital tract origin, Taiwan $J$ Obstet Gynecol 2010; 49:513-4.

7. Brander $\mathrm{JH}$, Buchnan MI, Rupture of the vagina during spontaneous delivery, Obst and Gyane. 1964;24:151-4.

8. Ramirez PT, Klemer DP, Vaginal evisceration after hysterectomy, $A$ literature review. Obstet Gynecol Surv 2002;57:462-7.

9. Cronin B, Sung VW, Matteson KA, Vaginal cuff dehiscence: Risk factors and management Am J Obstet Gynecol 2012;206:284-8.

10. Ostellng DL, Mc Creedy PA, MC Fee JG, Thompson HE, Prim. Complete colporasis, Obs and Gynae 1971:38,96-8 